\title{
Synergistic Effect between Sunshine Sports and Wisdom Sports
}

\author{
Feng Sun \\ College of physical education, Shandong University of Finance And Economics, Jinan Shandong 250014
}

Keywords: Sunshine Sports, Wisdom Sports, Synergy, Research

\begin{abstract}
In recent years, the voice of the reform of quality education is getting louder and louder. How to cultivate talents with comprehensive qualities is also an issue that people are paying increasing attention to. In this paper, we analyze the synergies between sports systems and other education subsystems in quality education and the optimization measures of the synergistic effects of physical education. Physical education can give full play to the synergistic effect between sunshine sports and wisdom sports, which can effectively improve the overall effectiveness of quality education.
\end{abstract}

\section{Introduction}

As an important component of quality education, physical education has been neglected by most educators and parents. However, the significance of physical education is undeniable. Physical education can not only improve people's physical quality and brain work efficiency, but also help people The cultivation of good moral character can be said that the benefits brought by sports are many, so it is necessary to develop physical education. Quality education contains the elements of moral education, aesthetic education, intellectual education and sports. They are distinguished from each other but are closely linked to each other. The synergistic effect of these major elements is exerted, which is an important task of improving the level of physical education and also the development of quality education important task.

December 23, 2006, in order to fully implement the party's education policy, conscientiously implement the "health first" guiding ideology among the hundreds of millions of students set off a wave of physical activity, and effectively improve the physical fitness of students, the Ministry of Education, the National Sports The Central Committee and the Communist Youth League Central Committee issued the Decision on Launching Sunshine Sports for Billions of Students across the Country. Since 2007, in line with the full implementation of the National Students' Constitution and Health Standards, the Central Government has extensively and deeply carry out the national billions of students Sunshine Sports. "So far, in school sports and more of a term" Sunshine Sports. "Now," Sunshine Sports "frequently appear in the media, but many people and even physical education teachers And students are quite strange to the "Sunshine Sports", I do not know the goals, content and the relationship with the school sports, these problems cannot be solved, will hinder the sunshine sports in schools.

The core content of quality education is to cultivate socialist builders and successors with all-round development in moral, intellectual and physical capacities. The realization of its goal is inseparable from the coordinated operation among various education subsystems in the system. Only by keeping the internal operation of each subsystem reasonable and promoting the coordination, cooperation, mutual penetration and mutual supplement of all subsystems to form a synergistic effect can we achieve better results improve the overall effectiveness of quality education.

Therefore, to understand the synergistic relationship between sports system and other education subsystems in quality education and make full use of the synergistic effect of sports is of great significance to the improvement of overall effectiveness of quality education. 


\section{The Proposed Methodology}

Connotation of synergies. In the 1960s Professor Hermann Haken, a professor of theoretical physics at the University of Stuttgart in the Federal Republic of Germany, summed up the common feature of the orderly structural formation of different phenomena in the study of lasers: an unbalanced system consists of a large number of sub- System, under certain conditions, the synergies and the coherence effects between the subsystems through the nonlinear coupling and interaction make the system form a self-organizing structure with certain functions, and macroscopically produce the time, space or space-time The orderly structure. It is pointed out that although the subsystems of different systems vary in their nature, they are macroscopically qualitatively changed. The mechanism from the old structure to the new structure is similar or the same, they all follow some common law.

The principle of synergistic effect means that in a complex large-scale system, the synergistic behaviors of all subsystems produce a single function that goes beyond the individual elements themselves to form a unified and synergistic effect of the entire system. This principle points out that the orderliness of the system is formed by the synergy of the various elements. Synergy is the inherent self-organizing ability of any complex system itself and the internal force that forms the systematic orderly structure.

Physical education is a subsystem of the quality education system. It has its own complete structure and operation mechanism. In addition to sports knowledge and sports skills, sports and ideological, moral and intellectual elements are also included in the structure and mechanism. The elements Interact with each other, interaction, it is impossible to completely peel them off. This fully reflects the existence of sports and other education in the co-operation between the relationships. The interaction between the sports system and other subsystems can effectively promote the formation of orderly state of the quality education system and improve the overall performance of the quality education.

As a subsystem of quality education, physical education itself has its own rules of operation. Under the influence of the structure of this education system, in addition to the sports knowledge and training methods necessary for itself, physical education still exists And moral education, aesthetic education, intellectual education of these educational factors fit point in physical education, should not be completely separated from each other should give full play to their synergies, through the whole system of physical education and other The synergistic effect between subsystems such as moral education, aesthetic education and intellectual education makes the physical education and the entire quality education be driven up and developed more and more rapidly.

Physical education is the precondition and foundation of the implementation of moral education. The human body is the foundation that carries all knowledge, skills, concepts and social awareness. Only a healthy body can serve as a carrier of thought and morality. It can be said that the premise of moral education is based on physical education of. At the same time, sports itself is infiltrating moral education all the time in the process of education. Specific performance for the following points: First, sports contribute to the formation of the firm will of students, sports training and the stimulation of the potential of human potential, require students to psychologically and physically overcome the difficulties, only perseverance Training and hard work to finalize the training content, sway in the game freely. Second, physical education is conducive to the cultivation of students' progressive consciousness. We all know that sports are competitive in nature. In some sporting events, in addition to superb sportsmanship and a healthy body, a proactive and victorious attitude is also necessary. Because of the development of sports ability is gradual and only solidly trained, stable attitude can achieve the established goal, which also helps students pragmatic, not quick success and good quality of development. Thirdly, PE is a particularly obvious subject of collectivization. Many of its sports programs require collective cooperation and team synergy. This is conducive to the formation of students' collective awareness and team concept, which enables them to fully realize their own value in the collective, and through the comparison with others, they can also more objectively understand and evaluate themselves. In addition, as a part of the group, students can also improve their 
organizational and discipline, so as to cultivate good interpersonal relationships indirectly with the teammates.

Sunshine sports diversified path. The goal of other sports education is to achieve the goal of achieving students' physical quality through education and learning of sports theory. On the basis of the traditional sports education mode, sunshine physical education pays more attention to the cultivation and improvement of students' physical quality, mental quality, physical and cultural qualities and lifelong physical ability, pay more attention to the participation of physical exercise, pay more attention to sports associations, sports and cultural festivals, sports clubs and other diversified higher physical education methods of use, which can effectively improve all aspects of the importance of physical education awareness, effectively improve college students to participate in sports learning and exercise initiative. Therefore, the higher physical education to sunshine sports oriented practical feasibility.

Sports synergy. Physical education and aesthetic education are two important subsystems under the education of quality. They differ from each other but are closely linked and influence each other. The two can greatly improve the efficiency and effectiveness of their own and overall quality education through coordinated actions.

Sports contribute to the formation of elegant body shape. In physical education, the use of correct and scientific teaching methods, and insisted that it is able to perfectly create a bodybuilding, well-proportioned body color, solid bones, smooth lines and strong muscles are Is the embodiment of human beauty, which makes people have a healthy body.

Intelligence is also the ability of people to perceive objective things and to play a subjective initiative to deal with and solve practical problems. The so-called intellectual education, that is, for the cultivation of people's intelligence and education. Briefly, intelligence is the comprehensive expression of our ideological and behavioral observations such as observation, memory, imagination, analysis and reasoning. We all know that the brain is the carrier of conscious thinking of people and is also the material undertaker of intelligence. The development of a person's intelligence is closely related to the health of the brain's owner. Related research shows that regular exercise can help brain cells grow and develop and make it more dynamic so as to be able to coordinate and command the body more sensitively and accurately, making work and study more efficient. Can be seen that the impact of physical education on human intelligence is enormous, the body often carry out physical activity can contribute to the development of intelligence, the same intellectual on physical education also has some negative effects. The use of scientific training methods and sports knowledge can improve athletes' training efficiency and athletic ability. So, the two are closely linked and interact with each other.

Physical exercise helps the growth of the brain. Related research shows that exercise can promote the development of the corresponding central brain, so that the central nervous system and cerebral cortex working ability to be improved, so that the flexibility and balance of the nerve and the working ability of the cerebral cortex are comprehensively improved.

Sports contribute to the recovery of human fatigue. At present, the pressures of study and work all the time haunt people. Long hours of study and work often make people tired. Generally speaking, continuous work and study for more than two hours reduce the work efficiency of the brain so much that Learning tasks cannot be fully completed. According to some studies, if proper physical exercise helps to improve the working efficiency of the brain when the brain is overloaded and tired, sports play an important role in daily working life.

Relationship between sunshine sports and school sports. From the academic point of view, the objectives of school physical education are to develop students' physical and psychological potential, enhance students' physique, enhance their health and promote the harmonious development of their physical and mental health; to develop students' attitudes, interests, habits and abilities to engage in physical exercise and lay the foundation for lifelong physical education Good foundation; promote individual socialization of students, develop good ideological and moral qualities of students so as to make them qualified personnel with the spirit of innovation and innovation and the moral, intellectual, and comprehensive development of socialism. The main ways to achieve this goal are physical 
education teaching, inter-class exercises, extra-curricular sports activities, after-school sports training and competition.

There is no contradiction between school sports and the development of sunshine sports. Both goals are consistent, all to enhance students' physical health, but sunshine sports more clarity, time constraints, quantitative standards. The two ways are the same, all relying on the teaching of physical education, extracurricular sports activities, but the Sunshine Sports pay more attention to the implementation of the "National Students 'Physical Health Standards" and compliance, but also pay more attention to the implementation of 1 hour of students' daily physical activity, More emphasis on extra-curricular sports activities. The requirements of both are the same. They all actively implement the education policy of the state, promote the quality education, conscientiously implement the guiding ideology of "health first", carry out the work in accordance with the requirements of the school sports work policy, and ensure the class time Teaching, to be equipped with strong physical education teachers, to extensively carry out extracurricular sports activities, to strengthen the school sports facilities and equipment to ensure that the school sports safety and so on. If we say that the difference between the two can be understood as such, school sports relies more on physical education, focusing on education, science and discipline to develop an all-round development of people; while the sun sports are based on the implementation of "National Students' Health Standards" as the basis , The main line around the goal of improving students' physical health in the short term, to carry out a wide range of extra-curricular activities, and actively implement the 1 hour a day as a means to achieve the common goal of school sports and sunshine sports. The focus of the two is not the same the introduction of sunshine sports can be said that it is in the past to strengthen the school physical education is relatively weak aspects. Therefore, Sunshine Sports and School Sports are not contradictory. The promotion of work between the two is not mutually exclusive. It does not mean that while conducting school sports work, it should also engage in a relatively independent set of sunshine sports.

\section{Conclusion}

Physical education and several other forms of education are important components of quality education. They are irreplaceable in themselves, but they are interrelated and mutually reinforcing. Therefore, how to maximize the synergistic effect of sports, moral education, aesthetic education and intellectual education so as to make the comprehensive and multi-faceted development of talents be the content that educators must study and consider. Only by fully exerting and using such synergistic effects of physical education can the quality education in our country be constantly perfected and developed. Sports and moral education, intellectual education and aesthetic education are conditions, interactions and coordinated development. The synergistic effect is a multi-level and all-round synergistic process that requires the joint efforts of the majority of the sports workers and relevant competent departments so as to ensure the cultivation of qualified personnel who meet the social needs of moral, intellectual and physical development.

\section{References}

[1]. Wang, L., Li, H. and Yang, L., 2017. Research on innovation system construction of physical education teaching mode in colleges and universities from the perspective of sunshine sports.

[2]. Wang, D., 2017. 38. Research on Fuzzy Comprehensive Evaluation of Sunshine Sports in Colleges and Universities. Revista de la Facultad de Ingeniería, 32(8).

[3]. Qian-li, G.A.O. and China, E., 2017. Three-factor Cultural Interaction Development Model Construction of Tertiary Sunshine Physical Education Program. Heilongjiang Researches on Higher Education, 7, p.031.

[4]. Sunshine, S.A., Goodman, R., Arya, M., Chu, L. and Ripberger, M., TixTrack Inc, 2017. Sports and concert event ticket pricing and visualization system. U.S. Patent Application 15/387,488. 